\title{
Retrospective analysis of video-instructed dispatcher-assisted cardiopulmonary resuscitation in Seoul, a metropolitan city
}

Hee Soon Lee

EMS Situation Management Center

Kicheol You

Hallym University College of Medicine

Jin Pyeong Jeon

Hallym University College of Medicine

Chulho Kim

Hallym University College of Medicine

Sungeun Kim ( $\nabla$ kimsueu1@hanmail.net )

EMS Situation Management Center

\section{Research Article}

Keywords: Out-of-hospital cardiac arrest, Cardiopulmonary resuscitation, Video-call

Posted Date: April 20th, 2021

DOI: https://doi.org/10.21203/rs.3.rs-413090/v1

License: (c) (i) This work is licensed under a Creative Commons Attribution 4.0 International License.

Read Full License

Version of Record: A version of this preprint was published at Scientific Reports on July 30th, 2021. See the published version at https://doi.org/10.1038/s41598-021-95077-5. 


\section{Abstract}

We aimed to investigate whether video-instructed dispatcher-assisted (DA)-cardiopulmonary resuscitation (CPR) improved neurologic recovery and survival to discharge compared to audio-instructed DA-CPR in adult out-of-hospital cardiac arrest (OHCA) patients in a metropolitan city with sufficient experience and facilities. A retrospective cohort study was conducted for adult bystander-witnessed OHCA patients administered DA-CPR due to presumed cardiac etiology between January 2018 and October 2019 in Seoul, Korea. The primary and secondary outcomes were the differences in favorable neurologic outcome and survival to discharge rates in adult OHCA patients in the two instruction groups. Binary logistic regression analysis was performed to identify the outcome predictors after DA-CPR. A total of 2109 adult OHCA patients with DA-CPR were enrolled. The number of video and audio instructed DA-CPR procedures was 387 (18.3\%) and 1722 (81.7\%), respectively. Elderly patients and those outside the home or medical facility were more likely to receive video instruction. Favorable neurologic outcome was observed more in patients who received video-instructed DA-CPR $(n=75,19.4 \%)$ than in patients who received audioinstructed DA-CPR $(n=117,6.8 \%)$. The survival to discharge rate was also higher in video-instructed DACPR $(n=105,27.1 \%)$ than in audio-instructed DA-CPR $(n=211,12.3 \%)$. Video-instructed DA-CPR was significantly associated with neurologic recovery $(\mathrm{aOR}=2.11,95 \% \mathrm{Cl}: 1.48-3.01)$ and survival to discharge (aOR $=1.81,95 \% \mathrm{Cl}: 1.33-2.46)$ compared to audio-instructed DA-CPR in adult OHCA patients. Video-instructed DA-CPR was associated with favorable outcomes in adult patients with OHCA in a metropolitan city equipped with sufficient experience and facilities.

\section{Background}

Out-of-hospital cardiac arrest (OHCA) is one of the leading causes of death and millions of people each year die from OHCA. In Korea, the number of out-of-hospital cardiac arrests is increasing every year with increases in population age and cardiovascular diseases. ${ }^{1}$ The incidence rate of OHCA was 37.5 per 100,000 population in 2006 and increased to 46.8 per 100,000 population in 2010 . Since cardiac arrest patients can be resuscitated within four minutes after proper treatment without neurologic complications, bystander cardiopulmonary resuscitation (CPR) is a key factor in improving the survival and neurologic recovery of patients with OHCA. ${ }^{2}$ Dispatcher-assisted CPR (DA-CPR) has been performed to improve the quality of bystander CPR. Song et al. ${ }^{3}$ reported that DA-CPR increased neurologic recovery rates $(2.0 \%$ in 2010 and 3.6\% in 2011) and discharge survival rates (7.1\% in 2010 and 9.4\% in 2011). In most cases of OHCA, DA-CPR has been carried out by audio instruction via a telephone call. ${ }^{4}$ Due to the nature of audio instruction, the patients' condition can only be identified through the caller's eye. Accordingly, concerns are raised during audio-instructed DA-CPR such as whether 1 ) it is a cardiac arrest situation, 2) CPR is being performed correctly, and 3 ) the patient's condition changed during CPR. In addition, the need for real-time feedback has increased in terms of the correct CPR position and rate. As mobile phone technology has developed, the number of video calls has increased in real life. ${ }^{5}$ Therefore, there has been an issue of whether audio or video instruction is more effective for DA-CPR in real life. Theoretically, video-instructed CPR allows for real-time feedback between the dispatcher and caller and better 
identification of the patient's condition than audio-instruction. ${ }^{6}$ A meta-analysis conducted by Lin et al. ${ }^{7}$ revealed that video-instructed DA-CPR provided significantly improved chest compression rates with more correct hand positions. Recently, Lee at al. ${ }^{5}$ reported early clinical experience with video-instructed DACPR and analyzed the survival outcomes compared to those from audio-instructed CPR in Seoul, a metropolitan city in Korea, between January and December in 2017 In their study, video-instructed DACPR was associated with a higher rate of survival to discharge and favorable neurologic outcomes via unadjusted analysis. However, this relationship was not statistically significant in the adjusted analysis. We postulated that the inclusion of 3-month trial period results and incorrect CPR posture may have affected the outcomes despite the instruction provided. Therefore, further study is required to evaluate the clinical efficacy of video-instructed DA-CPR under circumstances of ample experience and facilities. In this study, we aimed to investigate whether video-instructed DA-CPR resulted in improved neurologic recovery and survival to discharge compared to audio-instructed DA-CPR in a city setting.

\section{Results}

\section{Clinical characteristics of the patients}

A total of 5041 cases of OHCA assessed by EMS were collected after screening the initial data from the Seoul Emergency Operation Center. After excluding unknown, non-bystander-witnessed arrest cases, and EMS-witnessed arrests cases $(n=2648)$, presumed non-cardiac etiology $(n=256)$, and pediatric cases $(n=28), 2109$ cases were eligible for the final analysis (Figure 1). Among them, 387 patients $(18.3 \%)$ received video-instructed DA-PCR. The trends in the bimonthly incidence of DA-CPR are shown in Figure 2. The number of video-instructed DA-CPR procedures ranged from 22 and 53 every two months, which was lower than that of audio-instructed DA-CPR between 123 and 177 (Figure 2A).

The patients who received audio-instructed DA-CPR were older than those who received video-instructed DA-CPR $(p<0.0001)$. Elderly patients (> 65 years) and those outside the home or medical facility were more likely to be associated with the use of video-instructed DA-CPR $(p<0.0001)$ (Figure 3$)$.

\section{Outcome comparisons between video and audio-instructed DA-CPR}

The rate of favorable neurologic outcome was $19.4 \%$ (75 out of 387 ) in patients who received videoinstructed DA-CPR and 6.8\% (117 out of 1722) in those who received audio-instructed DA-CPR (Table 1). Throughout all periods, video-instructed DA-CPR resulted in more than $2.4 \%$ to $34.7 \%$ higher rates of neurologic recovery compared to audio-instructed DA-CPR (Figure 2B). Favorable neurologic outcome was associated with male gender ( $\mathrm{aOR}=1.92,95 \% \mathrm{Cl}$ : 1.26-2.92), coronary artery disease $(\mathrm{aOR}=1.85$, $95 \% \mathrm{Cl}: 1.23-2.78)$, other CPR locations (neither home nor medical or nursing institution) (aOR= 2.33, 95\% Cl: 1.63-3.32), and video instruction (aOR= 2.11, 95\% Cl: 1.48-3.01) (Table 2).

The rates of survival to discharge were $27.1 \%$ (105 out of 387 ) in video-instructed DA-CPR and $12.3 \%$ (211 out of 1722) in audio-instructed DA-CPR. Higher rates of survival to discharge was closely related to video instruction ( $\mathrm{aOR}=1.81,95 \% \mathrm{Cl}: 1.33-2.46)$ (Table 3$)$. Based on the above results, video-instructed 
DA-CPR was significantly associated with favorable neurologic outcome and survival to discharge compared to audio-instructed CPR.

\section{Discussion}

At the scene, the rapid recognition of cardiac arrest and subsequent CPR delivered in the right position were important in achieving a favorable prognosis in patients with OCHA. 8,9 In cases of impending cardiac arrest, witnesses often misinterpret patient movements as signs of life when they encounter agonal breathing and tremors. ${ }^{10}$ Accordingly, improvement in the initial CPR quality administered by a witness without delay at the scene is the main issue affecting the prognosis of OHCA patients.

Theoretically, a video call can be advantageous for providing information and a better understanding of the dispatchers compared to audio calls. Johnsen et al. ${ }^{6}$ reported that video-assisted DA-CPR allowed for appropriate feedback to the dispatchers and improved rescuer compliance in a simulation study. A metaanalysis ${ }^{7}$ also revealed that video instruction allowed for a faster chest compression rate (104.8 in video instruction vs 80.6 in audio instruction) and a lower risk of incorrect hand positioning. However, the delayed initiation of chest compression was observed in video-instructed DA-CPR compared to audioinstructed DA-CPR. In the real clinical field, there has been a concern about the delay of CPR initiation during the video call connection process due to the adjustment of the camera angle, change of the screen, and monitoring the patient's condition. To avoid delaying the CPR procedures at our institution, the first cycle of CPR was performed by audio instruction and then another caller was asked to switch to a video call. Although Lee at al. ${ }^{5}$ did not find significant differences in the time intervals between video and audio instruction ( $p=0.12$ ), further randomized controlled trials are necessary to identify the optimal protocol for video-instructed DA-CPR to obtain favorable neurological recovery and survival to discharge rates without a delay in initiating CPR.

The positive effects of video-instructed DA-CPR were demonstrated in a simulation study. However, the first real-world clinical study failed to prove the clinical efficacy of video-instructed DA-CPR on survival outcome. ${ }^{5}$ In the analysis conducted after adjusting for all covariates, video-instructed DA-CPR did not increase favorable neurologic outcomes $(\mathrm{OR}=1.28 ; 95 \% \mathrm{Cl}: 0.73-2.26)$ and survival to discharge rates (OR= 1.20; 95\% Cl: 0.74-1.94). ${ }^{5}$ However, that study investigated data from 2017, including the 3-month trial period results (Supplemental Figure S1). During the trial period between January to March in 2017, the favorable CPR outcome rates were lower in video-instructed DA-CPR than audio-instructed DA-CPR. When an emergency dispatcher gave CPR instructions using video calls during the trial period, the quality and characteristics of bystander CPR were different and inappropriate despite instructions outlined in the audio-instructed CPR protocol. To overcome these limitations, we transmitted the correct CPR position to the reporter's video screen during video-instructed DA-CPR (Supplemental Figure S2). Therefore, more patients with correct CPR positions may have been included in this study than in the previous study ${ }^{5}$ and this affected the favorable neurologic outcome and survival to discharge rates. 
Accumulated experience with video-instructed DA-CPR guidance can help enhance the effectiveness of the audio-instructed DA-CPR protocol. Video calls might not be possible depending on the caller considering the following conditions: 1) when caller was not good at using a mobile phone and could not be connected to a video call; 2) when the caller was confused and could not respond properly to the dispatcher's instructions; 3 ) when there was no other person in the vicinity other than one caller; and 4) when caller was connected to a video call, it was difficult to transmit information on the site because proper operation such as camera switching was not possible. In this case, feedback data obtained through video-instructed DA-CPR can be used to help the witness perform appropriate resuscitation during audio-instructed DA-CPR. In addition, the dispatcher provides proper management due to real-time feedback via the video-call until the EMS arrives at the scene. Professional first-aid treatment such as airway maintenance and the infusion of fluid or vasopressors can also be performed. Accordingly, a more effective linkage between pre-hospital and hospital treatment is expected through video calls.

There are some limitations to this study. First, this was a retrospective cohort study, although the number of CPR cases with video-instructed DA-CPR was the most to date. Accordingly, the potential risk of selection bias for video-instructed DA-CPR could affect the CPR outcomes. Second, CPR outcomes can be affected by various factors such as underlying medical diseases, the time from collapse to CPR, and the CPR location. Interestingly, the presence of coronary artery disease and the CPR location (neither home nor medical or nursing institution) was associated with favorable outcomes after CPR. The family of patients with coronary artery disease may be more likely to have appropriate CPR knowledge or less fear of performing CPR than those without family members with coronary artery disease. Regarding the CPR location, patients with advanced disease or underlying medical problems may be more likely to stay in medical institutions. Subgroup analysis according to CPR location was not performed in our investigation because we could not ascertain the severity of the underlying diseases in patients. Therefore, randomized controlled studies are required to accurately correlate video-instructed DA-CPR with the outcomes. Third, the differences in compliance with CPR instructions were not investigated in detail. In real life, CPR compliance as well as the instruction methods can be important factors influencing the outcomes. Accordingly, it is necessary to determine whether there is a difference in compliance according to CPR instruction methods in a subsequent study.

\section{Conclusion}

Video-instructed DA-CPR was associated with favorable outcomes in adult patients with OHCA in a metropolitan city equipped with sufficient experience and facilities. Randomized controlled trials are necessary to confirm our results.

\section{Methods}

\section{Emergency system in Seoul}


Seoul is a large city with a population of approximately 10 million people within a total of $605 \mathrm{~km}^{2}$. The number of 119 reports, the code indicating an emergency, per year is approximately 2 million, of which approximately 600,000 were disaster reports. ${ }^{5}$ To establish an effective disaster prevention system, the Seoul Emergency Operations Center (SEOC) was established in 2002. The SEOC is in charge of disasters such as typhoons, floods, heavy rain, strong wind, heavy snow, and earthquakes as well as emergency medical consultation and medical guidance in situations that threaten the safety and property of Seoul citizens. Currently, the system has been established with 24 fire departments and 151 emergency medical services. After the standard DA-CPR protocol is applied, ${ }^{5}$ a more systematic and immediate consultation is performed by professional medical staff. The emergency command system is operated by following a written protocol. First, when a call comes in, the primary situation call-taker receives the report and roughly ascertains the main contents of the report. Then, the call-taker confirms the chief complaint and delivers the dispatch order to each EMS. In particular, if emergency treatment is needed, including instruction after a pre-hospital cardiac arrest, a phone call to an emergency medical dispatcher is made immediately. The dispatcher ascertains the patient's condition and provides first aid guidance as necessary. In an out-of-hospital cardiac arrest, the secondary dispatcher immediately executes a dispatcher CPR order and shares information about the patient's condition with the emergency unit until they arrive at the scene. When the paramedics arrive, they contact to the first aid doctor in the center by phone, providing information on the patient's condition to the doctor and performing procedures according to the medical guidance provided (Supplemental Figure S3).

\section{Video-instructed DA-CPR}

This is a real-time support system of "Smart Video First Aid Instruction" where a 119 consultant provides first aid instructions through a video call with a caller until ambulance arrives. The system consists of three steps as follows: 1) after providing CPR instruction to the caller, the 119 consultant identifies the patient's condition through a video call; 2) the 119 consultant provides proper CPR techniques including the rescuer's position, hand position, and the adequate chest compression rate and depth until the emergency medical service (EMS) arrives; and 3) an emergency medicine doctor affiliated with the 119 situation room in SEOC constantly interacts with the EMS from the scene to the hospital. Video-instructed DA-CPR was attempted if the following conditions were met: 1 ) the presence of two or more bystanders and 2) the availability of a video call when CPR guidance based on the telephone consultation protocol was initiated by the secondary dispatcher. ${ }^{5}$ In such case, thee callers were reconnected by a video call. The system was conducted on a trial basis from January 16 to March 31, 2017. During the trial period, the system did not significantly increase the rates of favorable neurologic outcome and survival to discharge (Supplemental Figure S1). However, there were positive effects between the dispatcher and caller via video-instructed DA-CPR, such as real-time feedback, better identification of the patient's condition, and better guidance when the caller was hesitant or panicked in the emergency situation. Therefore, an official decision was made to implement the system by extending the period.

\section{Data collection, variables, and study outcomes}


We collected data from the EMS-assessed out-of-hospital cardiac arrest database in the SEOC between January 2018 and October 2019 (Figure 2). Records regarding demographical factors, underlying diseases, CPR location, and time from collapse to CPR were reviewed. The demographics included gender and age. Age over 65 years was used to define elderly patients. ${ }^{11}$ The underlying diseases were hypertension, diabetes mellitus, coronary artery disease, cerebrovascular disease, and cancer. The CPR location was divided into three groups of home, medical or nursing institutions, and other places. The time from collapse to CPR was categorized into four groups of time $<4 \mathrm{~min}, \geq 4$ and $<8 \mathrm{~min}, \geq 8$ and $<$ 15 min, and $>15$ min. $^{12}$

The primary outcome was the difference in favorable neurologic outcome in the video-instructed DA-CPR and audio-instructed DA-CPR. The favorable neurologic outcome was defined as cerebral performance category (CPC) scores of 1 and 2 measured at hospital discharge, indicating independent activities of daily life after CPR. ${ }^{12}$ The secondary outcome was the survival to discharge rates between the two instruction methods. We also compared the differences in the first rhythm recorded by the paramedics and the return of spontaneous circulation (ROSC) before arriving at the hospital. Study design was performed according to the principles of the Declaration of Helsinki and were approved by the Institutional Review Board (IRB) of the Chuncheon Sacred Heart Hospital (IRB No. 2020-09-016). All methods in this retrospective study were performed in accordance with the relevant guidelines and regulations in manuscript. The requirement for informed consent was waived by the IRB of the hospital.

\section{Statistical analysis}

Continuous variables are described as the mean and standard deviation (SD). A Chi-square or Student's ttest was performed to find differences according to the type of CPR instruction. Binary logistic regression analysis was performed to identify outcome predictors such as favorable neurologic outcome and survival to discharge in adult OHCA patients. Independent variables with a p value of $<0.05$ in univariable analyses were entered into multivariable models and expressed as adjusted odds ratios (aORs) with 95\% confidence intervals (Cls). Statistics were performed with R (version 3.6.1) and MedCalc (www.Medcalc.org) with a statistical significance indicated at $p<0.05$.

\section{Declarations}

\section{Acknowledgements}

None

\section{Author contributions}

SK designed and obtained funding for the study. HSL and JPJ were responsible for the data collection. $\mathrm{KY}$ and $\mathrm{CK}$ interpreted the data and performed statistical analyses. SK provided input for the final version of the manuscript. All authors reviewed the manuscript and provided editorial feedback. 


\section{Supplementary information accompanies this paper}

Conflicts of interest: All authors declare no competing interests.

Compliance with ethical standards: Data collection and study design were performed according to the principles of the Declaration of Helsinki and were approved by Coordinating Ethnics Committee of the Chuncheon Sacred Heart Hospital.

Data availability: Data are available from the corresponding author (SK) upon ethical approval from the IRB of the participating hospital.

\section{References}

1. Ro, Y.S. et al. A trend in epidemiology and outcomes of out-of-hospital cardiac arrest by urbanization level: A nationwide observational study from 2006 to 2010 in south korea. Resuscitation. 84, 547-557 (2013).

2. Perkins, G.D. et al. et al. Cardiac arrest and cardiopulmonary resuscitation outcome reports: Update of the utstein resuscitation registry templates for out-of-hospital cardiac arrest: A statement for healthcare professionals from a task force of the international liaison committee on resuscitation (american heart association, european resuscitation council, australian and new zealand council on resuscitation, heart and stroke foundation of canada, interamerican heart foundation, resuscitation council of southern africa, resuscitation council of asia); and the american heart association emergency cardiovascular care committee and the council on cardiopulmonary, critical care, perioperative and resuscitation. Resuscitation. 96, 328-340 (2015).

3. Song, K.J. et al. Dispatcher-assisted bystander cardiopulmonary resuscitation in a metropolitan city: A before-after population-based study. Resuscitation. 85,34-41(2014).

4. Lee, S.Y. et al. Recognition of out-of-hospital cardiac arrest during emergency calls and public awareness of cardiopulmonary resuscitation in communities: A multilevel analysis. Resuscitation. $128,106-111$ (2018).

5. Lee, S.Y. et al. Comparison of the effects of audio-instructed and video-instructed dispatcher-assisted cardiopulmonary resuscitation on resuscitation outcomes after out-of-hospital cardiac arrest. Resuscitation. 147, 12-20 (2020).

6. Johnsen, E., Bolle, S.R. To see or not to see-better dispatcher-assisted cpr with video-calls? A qualitative study based on simulated trials. Resuscitation. 78:320-326 (2008).

7. Lin, Y.Y. et al. Quality of audio-assisted versus video-assisted dispatcher-instructed bystander cardiopulmonary resuscitation: A systematic review and meta-analysis. Resuscitation. 123,77-85 (2018). 
8. Cummins, R.O. Emergency medical services and sudden cardiac arrest: The "chain of survival" concept. Annu Rev Public Health.14,313-333 (1993).

9. Dabrowska, A., Telec, W. [new guidelines of basic and advanced cardiopulmonary resuscitation and emergency cardiovascular care (ecc) american heart association (aha)]. Wiad Lek. 64, 127-131 (2011).

10. Eisenberg, M.S. Incidence and significance of gasping or agonal respirations in cardiac arrest patients. Curr Opin Crit Care. 12,204-206 (2006).

11. Nebsbjerg, M.A. et al. Skills among young and elderly laypersons during simulated dispatcher assisted cpr and after cpr training. Acta Anaesthesiol Scand. 62, 125-133 (2018).

12. Park, G.J.,et al. Timely bystander cpr improves outcomes despite longer ems times. Am J Emerg Med. 35, 1049-1055 (2017).

\section{Tables}

Table 1. Clinical characteristics of adult patients with OHCA according to the instruction method. 


\begin{tabular}{|c|c|c|c|}
\hline Variables & $\begin{array}{c}\text { Audio instruction } \\
(\mathrm{n}=1722)\end{array}$ & $\begin{array}{c}\text { Video instruction } \\
(\mathrm{n}=387)\end{array}$ & p-value \\
\hline \multicolumn{4}{|l|}{ Clinical findings } \\
\hline Female & $635(36.9 \%)$ & $124(32.0 \%)$ & 0.0734 \\
\hline $\begin{array}{l}\text { Average age }(\text { mean } \\
\text { years }\end{array}$ & $72.2 \pm 14.7$ & $64.9 \pm 16.2$ & \\
\hline Elderly $(\geq 65 y r)$ & $1260(73.2 \%)$ & $214(55.3 \%)$ & $<0.0001$ \\
\hline Hypertension & $616(35.8 \%)$ & $127(32.8 \%)$ & 0.2715 \\
\hline Diabetes mellitus & $419(24.3 \%)$ & $81(20.9 \%)$ & 0.1551 \\
\hline Coronary artery disease & $328(19.0 \%)$ & $71(18.3 \%)$ & 0.7503 \\
\hline Cerebrovascular disease & $184(10.7 \%)$ & $33(8.5 \%)$ & 0.2068 \\
\hline Cancer & $245(14.2 \%)$ & $54(14.0 \%)$ & 0.8889 \\
\hline CPR location & & & $<0.0001$ \\
\hline Home & $1239(72.0 \%)$ & $240(62.0 \%)$ & \\
\hline Medical/nursing institution & $184(10.7 \%)$ & $21(5.4 \%)$ & \\
\hline Other places & $299(17.4 \%)$ & $126(32.6 \%)$ & \\
\hline Time from collapse to CPR & & & 0.0640 \\
\hline$<4 \min$ & $80(4.6 \%)$ & $14(3.6 \%)$ & \\
\hline$\geq 4$ to $<8 \mathrm{~min}$ & $473(27.5 \%)$ & $122(31.5 \%)$ & \\
\hline$\geq 8$ to $<15$ min & $845(49.1 \%)$ & $197(50.9 \%)$ & \\
\hline$\geq 15 \mathrm{~min}$ & $324(18.8 \%)$ & $54(14.0 \%)$ & \\
\hline \multicolumn{4}{|l|}{ Outcome } \\
\hline First rhythm of paramedics & & & $<0.0001$ \\
\hline Asystole & $869(50.5 \%)$ & $140(36.2 \%)$ & \\
\hline PEA & $494(28.7 \%)$ & $94(24.3 \%)$ & \\
\hline $\mathrm{VT} / \mathrm{VF}$ & 359 (20.8\%) & $153(39.5 \%)$ & \\
\hline Out-of-hospital ROSC & $239(13.9 \%)$ & $115(29.7 \%)$ & $<0.0001$ \\
\hline Survival to discharge & $211(12.3 \%)$ & $105(27.1 \%)$ & $<0.0001$ \\
\hline $\begin{array}{c}\text { Favorable neurologic } \\
\text { outcome }\end{array}$ & $117(6.8 \%)$ & $75(19.4 \%)$ & $<0.0001$ \\
\hline
\end{tabular}

PEA, pulseless electrical activity; ROSC, return of spontaneous circulation; SD, standard deviation; VF, ventricular fibrillation; VT, ventricular tachycardia

a Data are shown as the numbers of subjects (percentage) for discrete and categorical variables and the mean \pm standard deviation.

Table 2. Binary logistic regression analysis of the adjusted odds ratio of the favorable neurologic outcome in adult patients with OHCA. 


\begin{tabular}{ccccc}
\hline Variable & \multicolumn{2}{c}{ Univariable model } & \multicolumn{2}{c}{ Multivariable model } \\
& OR $(95 \% \mathrm{CI})$ & $\mathrm{p}$-value & $\mathrm{aOR}(95 \% \mathrm{CI})$ & $\mathrm{p}$-value \\
\hline Older age (yes vs. no) & $0.13(0.10-0.19$ & $<0.001$ & $0.19(0.13-0.27)$ & $<0.001$ \\
Gender (male vs. female) & $3.05(2.07-4.51)$ & $<0.001$ & $1.92(1.26-2.92)$ & $<0.003$ \\
Time from collapse to CPR & & & & \\
$<4 \mathrm{~min}$ & 1.0 (reference) & - & 1.0 (reference) & - \\
$\geq 4$ to $<8 \mathrm{~min}$ & $1.27(0.65-2.49)$ & 0.477 & $1.10(0.53-2.31)$ & 0.792 \\
$\geq 8$ to $<15 \mathrm{~min}$ & $0.66(0.34-1.29)$ & 0.225 & $0.63(0.30-1.31)$ & 0.213 \\
$\geq 15 \mathrm{~min}$ & $0.23(0.10-0.54)$ & $<0.001$ & $0.26(0.10-0.67)$ & 0.005 \\
Underlying disease & & & & \\
Hypertension & $0.72(0.52-0.99)$ & 0.046 & $1.36(0.92-2.01)$ & 0.123 \\
Diabetes mellitus & $0.45(0.29-0.70)$ & $<0.001$ & $0.57(0.35-0.93)$ & 0.025 \\
Coronary artery disease & $1.35(0.95-1.92)$ & 0.095 & $1.85(1.23-2.78)$ & 0.003 \\
Cerebrovascular disease & $0.36(0.17-0.73)$ & 0.005 & $0.65(0.31-1.39)$ & 0.271 \\
$\quad$ Cancer & $0.24(0.12-0.50)$ & $<0.001$ & $0.41(0.19-0.87)$ & 0.020 \\
CPR location & & & & \\
Home & $1.0($ reference) & - & $1.0($ reference) & - \\
Others & $4.56(3.34-6.23)$ & $<0.001$ & $2.33(1.63-3.32)$ & $<0.001$ \\
Medical/nursing institution & $0.39(0.15-0.96)$ & 0.041 & $0.52(0.20-1.32)$ & 0.169 \\
Type of DA-CPR & & & & \\
Audio instruction & $1.0($ reference) & - & 1.0 (reference) & - \\
Video instruction & $3.30(2.41-4.52)$ & $<0.001$ & $2.11(1.48-3.01)$ & $<0.001$ \\
\hline
\end{tabular}

OR, odd ratio; aOR, adjusted OR; CI, confidence interval

Table 3. Binary logistic regression analysis of the adjusted odds ratio of the survival to discharge in adult patients with OHCA. 


\begin{tabular}{ccccc}
\hline Variable & \multicolumn{2}{c}{ Univariable model } & \multicolumn{2}{c}{ Multivariable model } \\
& OR $(95 \% \mathrm{CI})$ & $\mathrm{p}$-value & aOR $(95 \% \mathrm{CI})$ & $\mathrm{p}$-value \\
\hline Older age (yes vs. no) & $0.17(0.13-0.22)$ & $<0.001$ & $0.22(0.17-0.30)$ & $<0.001$ \\
Gender (male vs. female) & $2.55(1.90-3.41)$ & $<0.001$ & $1.77(1.28-2.44)$ & $<0.003$ \\
Time from collapse to CPR & & & & \\
$<4$ min & 1.0 (reference) & - & 1.0 (reference) & - \\
$\geq 4$ to $<8$ min & $1.38(0.79-2.41)$ & 0.258 & $1.31(0.71-2.43)$ & 0.390 \\
$\geq 8$ to $<15$ min & $0.69(0.39-1.19)$ & 0.183 & $0.70(0.38-1.29)$ & 0.255 \\
$\geq 15$ min & $0.30(0.15-0.58)$ & $<0.001$ & $0.34(0.17-0.72)$ & 0.005 \\
Underlying disease & & & & \\
Hypertension & $0.65(0.50-0.84)$ & 0.001 & $1.10(0.80-1.51)$ & 0.568 \\
Diabetes mellitus & $0.47(0.34-0.66)$ & $<0.001$ & $0.60(0.41-0.88)$ & 0.009 \\
Coronary artery disease & $1.41(1.06-1.88)$ & 0.018 & $1.97(1.41-2.75)$ & $<0.001$ \\
Cerebrovascular disease & $0.42(0.25-0.71)$ & 0.001 & $0.69(0.39-1.20)$ & 0.189 \\
Cancer & $0.21(0.11-0.37)$ & $<0.001$ & $0.31(0.17-0.58)$ & $<0.001$ \\
CPR location & & & & \\
Home & $1.0($ reference) & - & $1.0($ reference) & - \\
Others & $4.60(3.54-5.98)$ & $<0.001$ & $2.50(1.85-3.38)$ & $<0.001$ \\
Medical/nursing institution & $0.90(0.54-1.48)$ & 0.675 & $1.15(0.67-1.98)$ & 0.603 \\
Type of DA-CPR & & & & \\
Audio instruction & $1.0($ reference) & - & 1.0 (reference) & - \\
Video instruction & $2.67(2.04-3.48)$ & $<0.001$ & $1.81(1.33-2.46)$ & $<0.001$ \\
\hline
\end{tabular}

OR, odd ratio; aOR, adjusted OR; CI, confidence interval

Figures 


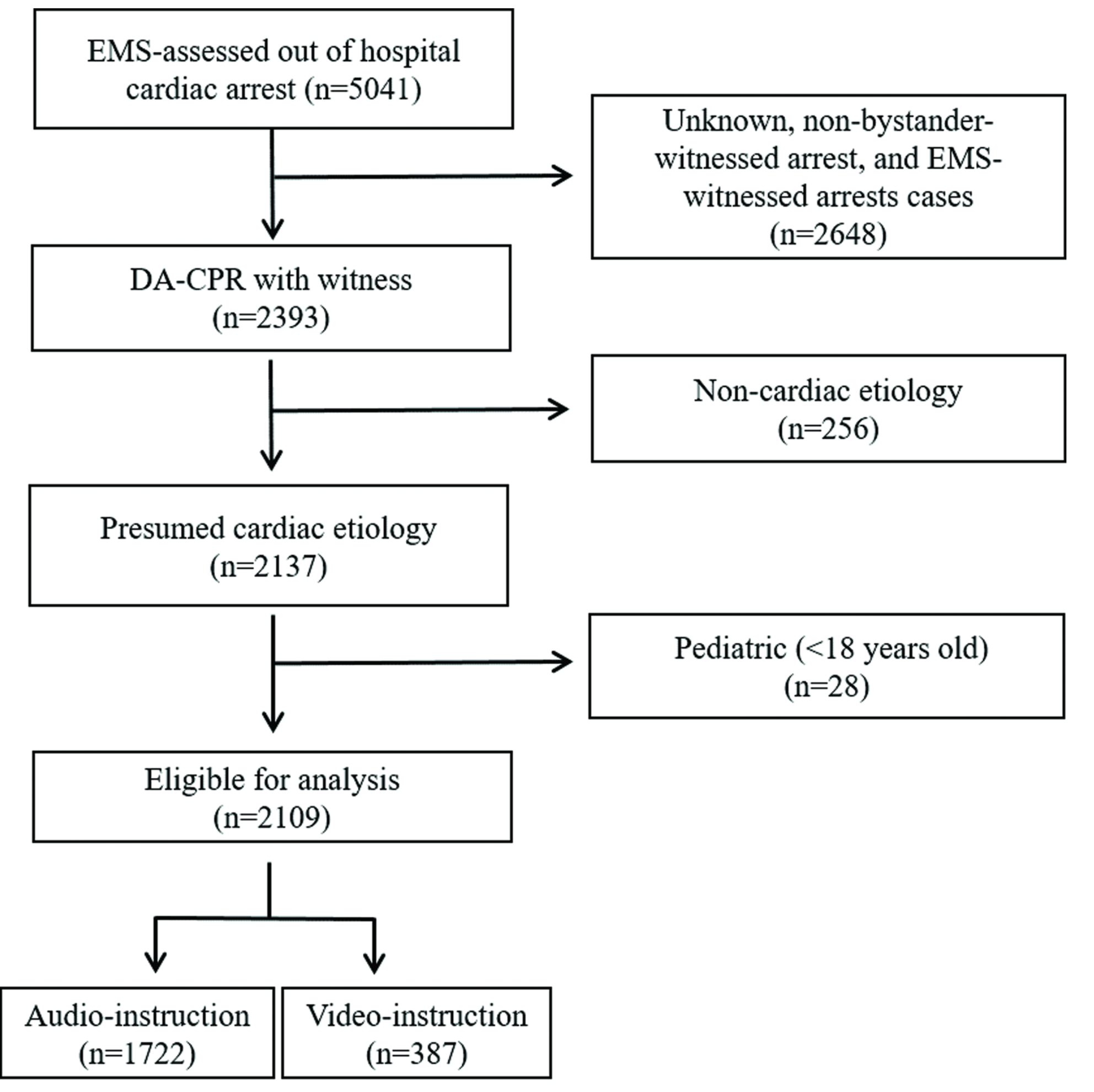

Figure 1

Flow diagram of the study population. EMS; emergency mdical service; DA-CPR, dispatcher-assisted cardiopulmonary resuscitation 


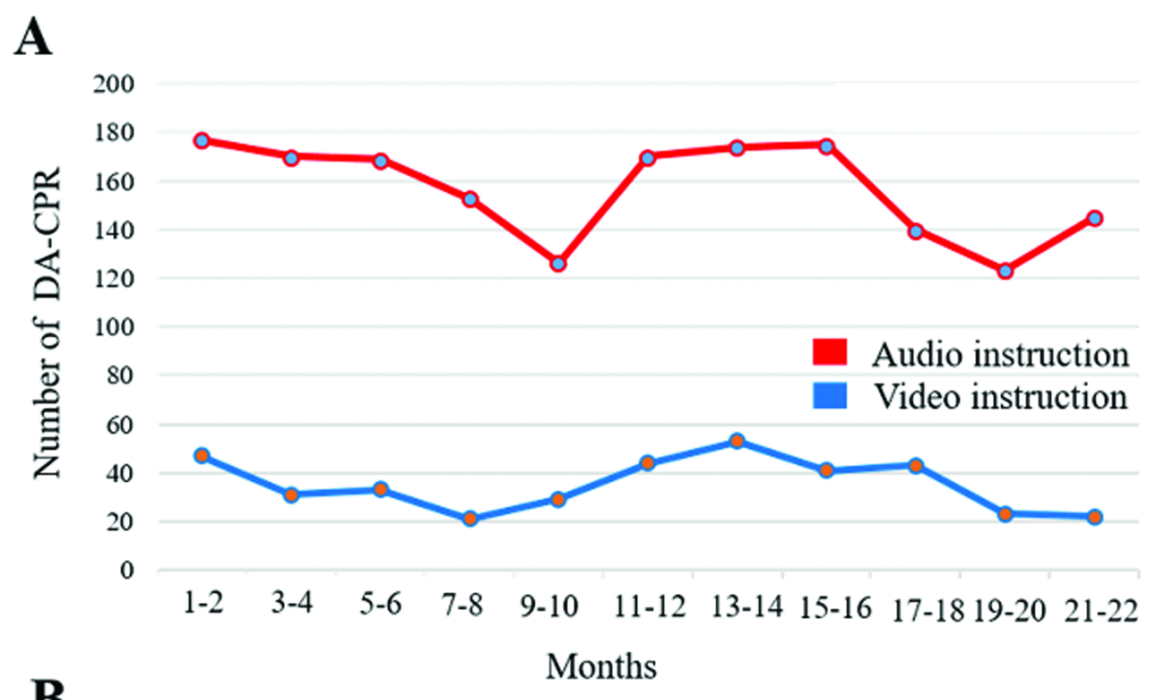

B

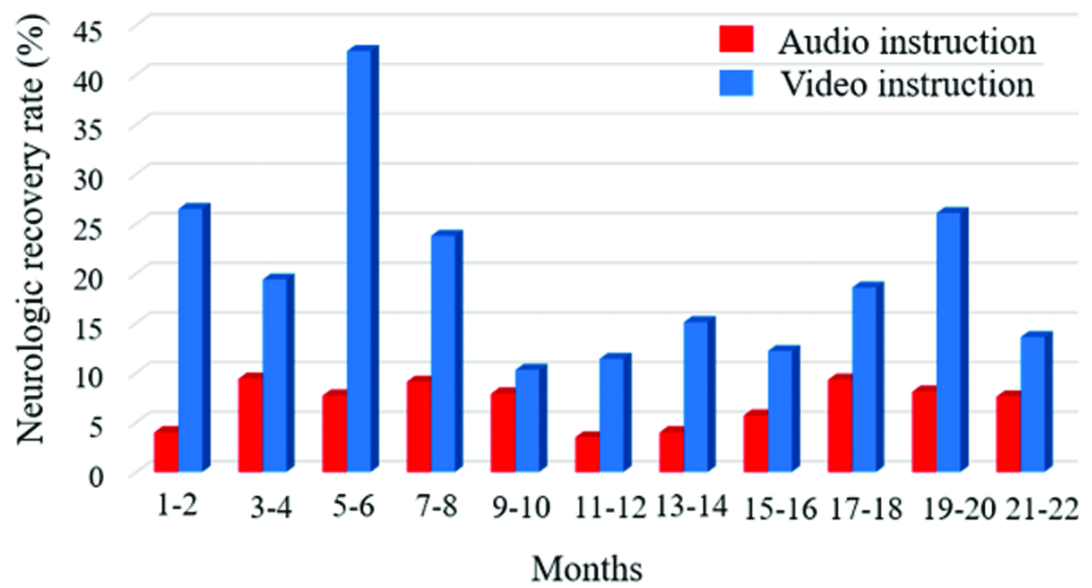

C

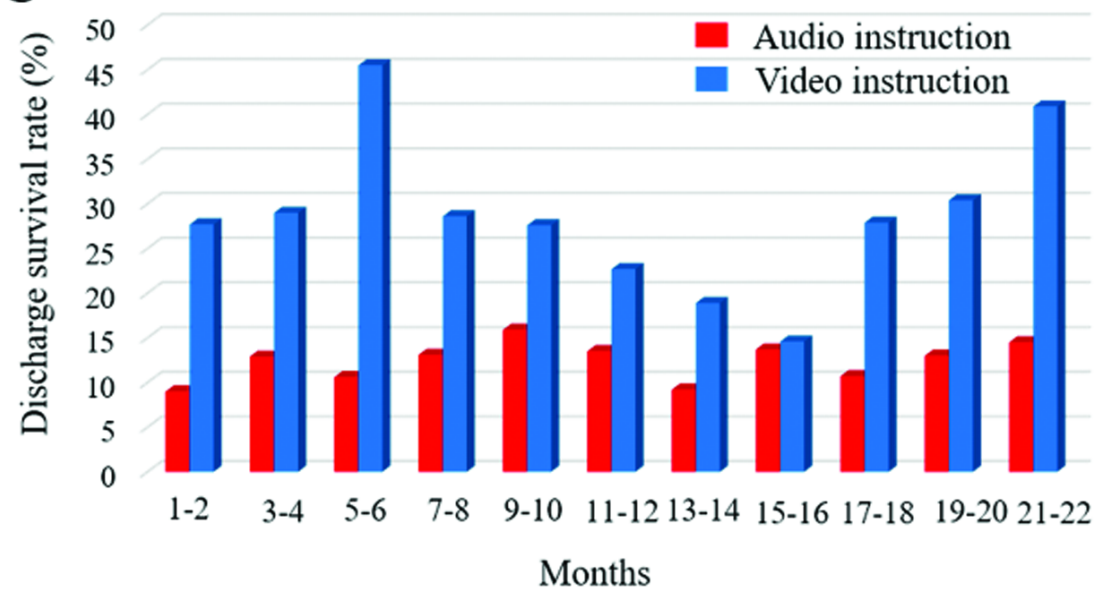

Figure 2

Trends in the bimonthly incidence of DA-CPR for adult patients with OHCA (A). Rates of favorable neurologic outcome (B) and survival to discharge (C) according to the CPR instructions delivered by video and audio-instructed DA CPR. 


\begin{tabular}{|c|c|c|c|c|}
\hline Variable & & Odds ratio & & p \\
\hline Time from collapse to CPR & $<4$ & & Reference & \\
\hline \multirow[t]{3}{*}{ minute } & $4-8$ & & $1.56(0.87,2.99)$ & 0.16 \\
\hline & $8-15$ & & $1.52(0.86,2.88)$ & 0.17 \\
\hline & $\geq 15$ & & $1.18(0.63,2.34)$ & 0.62 \\
\hline \multirow[t]{2}{*}{ Gender } & Female & & Reference & \\
\hline & Male & & $0.96(0.75,1.24)$ & 0.77 \\
\hline \multirow[t]{2}{*}{ Older age ( $\geq 65$ years) } & No & & Reference & \\
\hline & Yes & - & $0.51(0.40,0.65)$ & $<0.001$ \\
\hline \multirow[t]{2}{*}{ Hypertension } & No & & Reference & \\
\hline & Yes & & $1.13(0.87,1.46)$ & 0.37 \\
\hline \multirow[t]{2}{*}{ Diabetes } & No & & Reference & \\
\hline & Yes & & $0.95(0.70,1.26)$ & 0.71 \\
\hline \multirow[t]{2}{*}{ Cardiovascular disease } & No & & Reference & \\
\hline & Yes & & $1.05(0.78,1.41)$ & 0.73 \\
\hline \multirow[t]{2}{*}{ Cerebrovascular disease } & No & & Reference & \\
\hline & Yes & & $1.01(0.67,1.49)$ & 0.95 \\
\hline \multirow[t]{2}{*}{ Cancer } & No & & Reference & \\
\hline & Yes & & $1.24(0.88,1.73)$ & 0.20 \\
\hline \multirow[t]{3}{*}{ CPR location } & Home & & Reference & \\
\hline & Med/Nur ${ }^{\star}$ & $\longmapsto$ & $0.65(0.39,1.03)$ & 0.08 \\
\hline & Others & & $1.98(1.50,2.60)$ & $<0.001$ \\
\hline (Intercept) & & $\longmapsto$ & $0.20(0.10,0.38)$ & $<0.001$ \\
\hline
\end{tabular}

* Indicates medical or nursing institutions

\section{Figure 3}

Factors influencing video-instructed DA-CPR in adult patients with OHCA.

\section{Supplementary Files}

This is a list of supplementary files associated with this preprint. Click to download.

- Table12and3.docx

- SupplementalDataScireport.docx

- SupplementalDataScireport.docx 